\title{
Anti-CCL3 autoantibodies are not markers of type 1 diabetes when measured by a commercial ELISA method
}

\author{
A. G. Ziegler • U. Mollenhauer • P. Achenbach • \\ E. Bonifacio
}

Received: 12 August 2010 /Accepted: 26 October 2010 /Published online: 3 December 2010

(C) Springer-Verlag 2010

Keywords Autoantibody CCL3 - ELISA · Type 1 diabetes

\begin{abstract}
Abbreviations
AU Arbitrary units

IQR Interquartile range
\end{abstract}

Autoantibodies are a hallmark feature of type 1 diabetes and have proved useful markers for disease prediction and classification [1]. New autoantibodies are of interest since they may strengthen disease relevance in autoantibodypositive individuals [2, 3]. Recently, autoantibodies against the inducible chemokine CCL3 were reported to be associated with type 1 diabetes [4], and commercial kits for the measurement of anti-CCL3 autoantibodies are now available. CCL3 is produced during inflammation and is suggested to be produced by beta cells under stress. We had the opportunity to evaluate this commercial kit and here report our findings. Sera from patients with new or recent onset of type 1 diabetes ( $n=54$; median age 10 years; IQR $7.5-13.5$ years), controls ( $n=40$; median age 11.2 years; IQR 4.7-16.3 years), firstdegree relatives of patients with type 1 diabetes $(n=33$; median age 10.5 years; IQR 7.7-11.2 years) and participants with other autoimmune diseases $(n=17$; median age

\footnotetext{
A. G. Ziegler $(\bowtie) \cdot$ P. Achenbach

Institut für Diabetesforschung, Helmholtz Zentrum München, Ingolstaedter Landstr. 1,

85764, Neuherberg, Germany

e-mail: anziegler@1rz.uni-muenchen.de
}

\author{
A. G. Ziegler $\cdot$ U. Mollenhauer $\cdot$ P. Achenbach \\ Neuherberg, Germany \\ E. Bonifacio \\ Center for Regenerative Therapies Dresden, \\ Dresden University of Technology, \\ Dresden, Germany
}

Forschergruppe Diabetes e.V. am Helmholtz Zentrum München,
12.5 years; IQR 10.1-16.6 years) were tested with the Micromedic Technologies kit (MT-CCL3-IFU; Micromedic Technologies, Ramat Gan, Israel) according to the manufacturer's instructions. All sera had been stored at $-20^{\circ} \mathrm{C}$ prior to testing. One kit batch was used for all anti-CCL3 autoantibody measurements. The study participants gave informed consent, and the investigations were carried out in accordance with the Declaration of Helsinki as revised in 2000.

We found no association between anti-CCL3 autoantibodies and type 1 diabetes (Fig. 1). Instead, we found that the level of CCL3 autoantibodies correlated with the length of storage of the serum sample assayed. Using the threshold for positivity provided by the manufacturer, 24 of the 40 $(60 \%)$ controls, 17 of the $54(31 \%)$ type 1 diabetic patients, 19 of the $33(58 \%)$ first-degree relatives of type 1 diabetic patients, and 16 out of 17 (94\%) of the patients with other autoimmune diseases were positive for anti-CCL3 autoantibodies. Samples that were older than 5 years had markedly higher values than the recently collected samples (mean 414 arbitrary units [AU] vs $208 \mathrm{AU} ; p=10^{-13}$ ) and were more frequently positive $\left(81 \%\right.$ vs $\left.36 \% ; p=10^{-7}\right)$. It is possible, therefore, that the degradation of proteins, including serum CCL3, may in some way affect the assay. Comparison of recently collected samples from controls and patients showed no difference in anti-CCL3 autoantibody concentrations (161 AU vs $179 \mathrm{AU} ; p=0.56$ ). Thus, we cannot confirm the manufacturer's claim that the kit is useful as an aid to the diagnosis of type 1 diabetes. The original findings of anti-CCL3 autoantibodies were based on a fluid-phase radioassay, whereas the commercial kit is an ELISA. Hence, our evaluation of the performance of the kit does not invalidate the original findings. It is, however, important to note the relatively strong relationship with the duration of storage of sample, and in the original findings there is no mention of whether the control samples were collected at a similar time period as the patient samples. In 
Fig. 1 Anti-CCL3 autoantibody titres in healthy control children, children with newly diagnosed type 1 diabetes (T1D), firstdegree relatives (FDRs) of children with type 1 diabetes, and children with other autoimmune diseases. Means are indicated. The dashed line represents the threshold for positivity provided by the manufacture. Anti-CCL3 autoantibody titres were higher in serum samples stored at $-20^{\circ} \mathrm{C}$ for $>5$ years than in recently stored samples $\left(p=10^{-13}\right)$

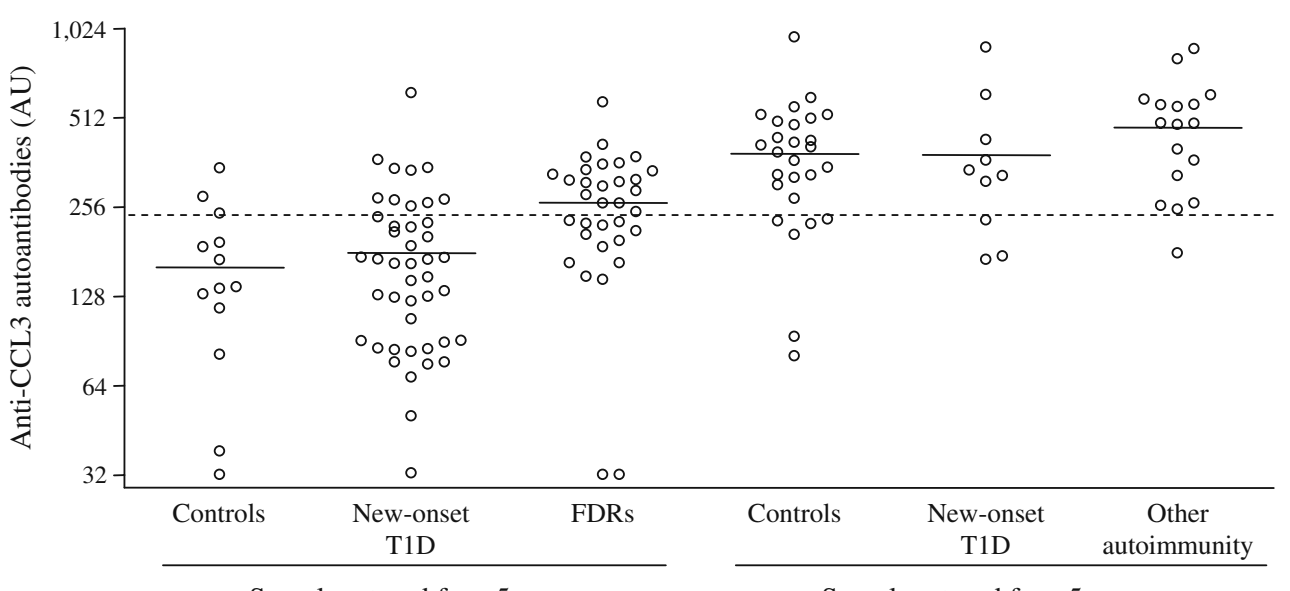

Samples stored for $<5$ years
Samples stored for $>5$ years conclusion, we would not recommend that the ELISAbased anti-CCL3 autoantibody kit be used to aid the prediction or diagnosis of type 1 diabetes.

Acknowledgements This study was supported in part by grants from the German Federal Ministry of Education and Research to the Competence Network for Diabetes mellitus (FKZ 01GI0805-07), the Deutsche Forschungsgemeinschaft (ZI 310/12-6), and the Juvenile Diabetes Research Foundation (P.A. 11-2005-1117). The Micromedic Technologies kits were kindly provided by M. Becker, Genzyme Virotech, Rüsselsheim, Germany.

Duality of interest The authors declare that there is no duality of interest associated with this manuscript.

\section{References}

1. Ziegler AG, Nepom J (2010) Prediction and pathogenesis in type 1 diabetes. Immunity 32:468-478

2. Wenzlau JM, Juhl K, Yu L et al (2007) The cation efflux transporter $\mathrm{ZnT} 8$ (Slc30A8) is a major autoantigen in human type 1 diabetes. Proc Natl Acad Sci USA 104:17040-17045

3. Achenbach P, Lampasona V, Landherr U et al (2009) Autoantibodies to zinc transporter 8 and SLC30A8 genotype stratify type 1 diabetes risk. Diabetologia 52:1881-1888

4. Shehadeh N, Pollack S, Wildbaum G et al (2009) Selective autoantibody production against CCL3 is associated with human type 1 diabetes mellitus and serves as a novel biomarker for its diagnosis. J Immunol 182:8104-8109 